\title{
The Mathematics of Joan Birman
}

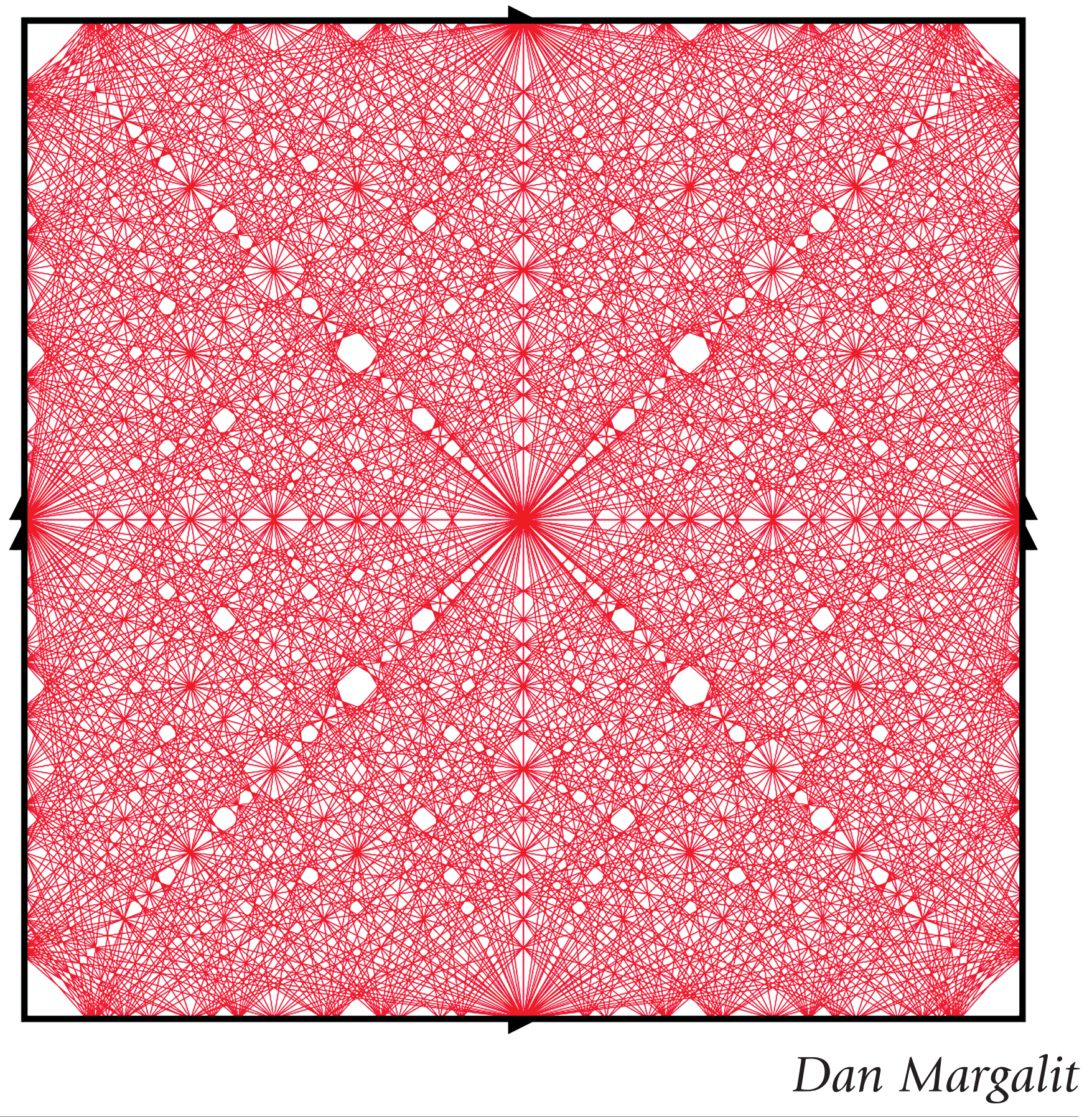

Dan Margalit is a Professor in the School of Mathematics at the Georgia Institute of Technology. His email address is margalit@math.gatech. edu.
He is supported by the National Science Foundation under Grant No. DMS 1057874. 


\section{Introduction}

Joan Birman published her first paper, "On braid groups," in January 1969. That work introduced one of the most important tools in the study of braids and surfaces, now called the Birman exact sequence. Fifty years and more than one hundred papers later, Birman is an active researcher and has long been established as a leading figure in the field of low-dimensional topology.

The goal of this article is to give a broad overview of Birman's mathematics. In the process, we will see several related themes emerge. Time and again, Birman has shown a knack for asking the right questions, for pursuing and embracing unlikely collaborations across mathematical disciplines, and for uncovering and revitalizing hidden or forgotten fields. Because of this, her work has often been ahead of its time, with important implications and applications found years or decades after the original discoveries. For instance, her book on braids is credited with bringing that theory from the fringes to the fore. Similarly, when Birman began working on mapping class groups and Torelli groups, she was working in isolation. Now these are core topics in topology, and her contributions are of fundamental importance. In fact, Birman's work has underpinned two Fields medals.

Birman's research revolves around the theories of knots, braids, mapping class groups of surfaces, and 3-manifolds. Figure 1 shows a diagram of these topics and gives a road map for this article. We will introduce the various objects and the connections between them in the sections indicated. It is a bit of a miracle that these subjects are so closely intertwined. In what follows we will see how Birman's work has influenced and interacted with this beautiful circle of ideas.

\section{$\S 1$ Knots}

A knot is the image of a smooth embedding of the circle $S^{1}$ into $\mathbb{R}^{3}$. We can think of a knot as a piece of string with its ends glued together. We can draw a diagram of a knot by projecting it to a plane and indicating the over/undercrossings of the strands by putting a break in the strand that is crossing below; see Figure 2. Two knots are equivalent if they are isotopic, that is, if one knot can be continuously deformed into the other without creating any selfintersections along the way.

The fundamental problem in knot theory is to decide if two knots are equivalent. A (not really) simpler version is to decide if a given knot is equivalent to the trivial knot. The knots in Figure 2 fall into two equivalence classes (left and right trefoils). Which are equivalent?

For permission to reprint this article, please contact:

reprint-permission@ams.org.

DOI: https://doi.org/10.1090/noti/1808

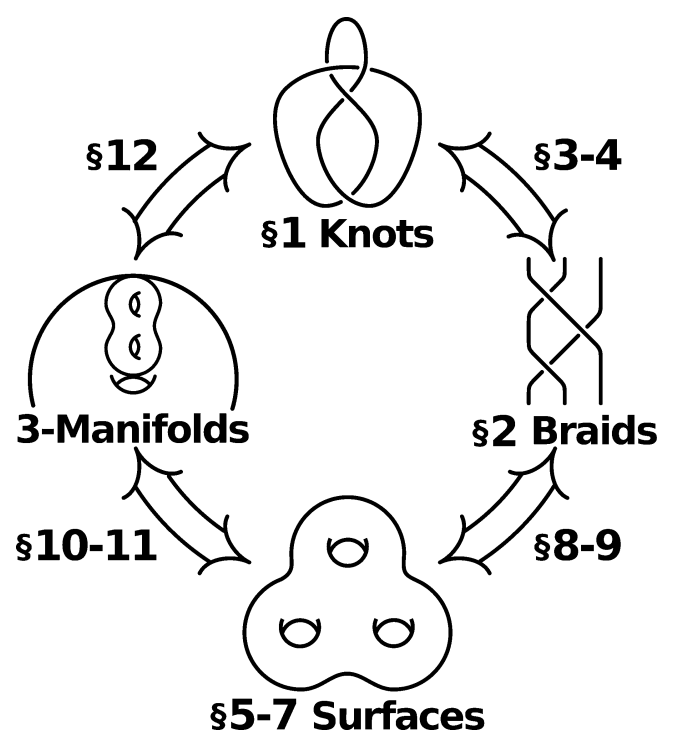

Figure 1. A road map for this article (and Birman's career).
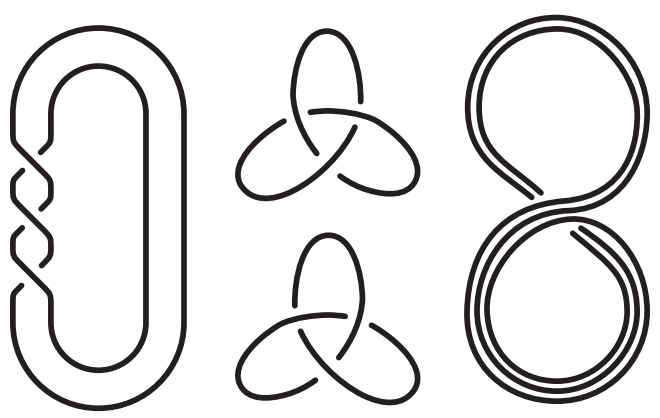

Figure 2. Some examples of knots.

As this exercise illustrates, knot theory is difficult because there are many diagrams for the same knot that are very different from one another. There is no easy way to move between two different diagrams, and there is no systematic way to choose a canonical diagram for a knot.

Among the many successes of knot theory is the discovery of knot invariants. An invariant for a knot is an object (number, polynomial, etc.) we can associate to a knot with the property that equivalent knots have the same invariant. If we find two knots with different invariants, then they are inequivalent knots.

One of the most famous and important knot invariants is the Alexander polynomial, a Laurent polynomial that can be computed from any knot diagram. The Alexander polynomial is not a complete invariant: it attains the same value on the left- and right-handed trefoil knots, and also Kinoshita and Terasaka found a nontrivial knot with the same Alexander polynomial as the trivial knot. The simplest diagram for the latter has 11 crossings. It is still an open problem to find an easily computable, complete invariant for knots (more on this later). 
Knot theory has applications to statistical mechanics, molecular biology, and chemistry; see Murasugi's book [71] for a survey. Later in this article we will see several connections of knot theory with other parts of topology, group theory, dynamics, and number theory.

\section{$\S 2$ Braids}

A braid on $n$ strands is a collection of $n$ disjoint paths in $\mathbb{R}^{2} \times[0,1]$, connecting $n$ points in $\mathbb{R}^{2} \times\{0\}$ to the corresponding points in $\mathbb{R}^{2} \times\{1\}$, and intersecting each plane $\mathbb{R}^{2} \times\{t\}$ in exactly $n$ points. The $n$ paths are called the strands of the braid.

We consider two braids to be equivalent if they are isotopic, that is, if we can continuously deform one to the other while holding the endpoints fixed and without allowing strands to pass through each other. Figure 3 shows two equivalent braids. The set of braids on $n$ strands forms a group $B_{n}$, with the group operation given by stacking braids.
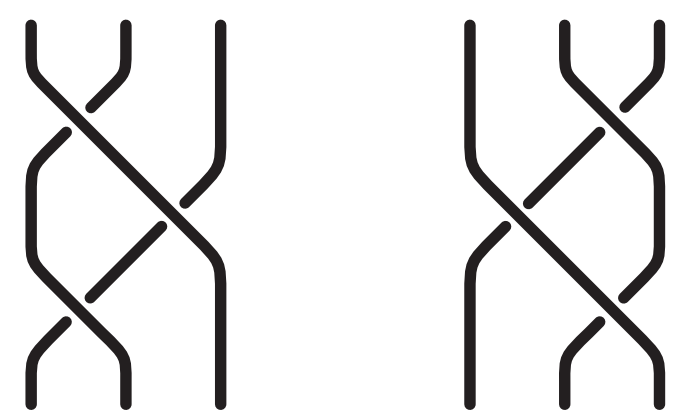

Figure 3. Two equivalent braids.

There is a more succinct (and sophisticated) way to define the braid group. Let $C_{n}$ denote the configuration space of $n$ distinct points in the plane. We have

$$
B_{n} \cong \pi_{1}\left(C_{n}\right)
$$

The isomorphism is obtained as follows. Let eta be a braid on $n$ strands. For each $t$ in $[0,1]$ we may consider the corresponding plane parallel to the original two planes. If we intersect this plane with the braid $e t a$, we obtain a point in $C_{n}$. As $t$ changes from 0 to 1 , we obtain a loop in $C_{n}$, that is, an element of $\pi_{1}\left(C_{n}\right)$. This map is the desired isomorphism.

We can now see why the braid group is ubiquitous in mathematics and science: it records the motions of points in the plane. The points can be roots of polynomials, critical values of branched covers, particles in a two-dimensional medium, or autonomous vehicles moving through city streets. See the survey by Birman and her student Brendle for an excellent introduction to the theory [16].

\section{$\S 3$ Braids and Knots}

There is a simple way to obtain a knot from a braid, namely by connecting the top of the braid to the bottom by $n$ parallel strands. Actually, in general we obtain a link, which is a disjoint union of knots. The resulting knot or link is called a closed braid; see the left-hand side of Figure 4 for an example. In 1923 Alexander proved the remarkable theorem that every knot is equivalent to a closed braid [3].

On the face of it, braids are more tractable than knots because of the group structure, and Alexander's theorem gives us hope of applying our knowledge of braid groups to the theory of knots. The immediate problem is that there are many braids giving rise to the same knot. For instance, if two braids are conjugate, then their braid closures are equivalent.

There are also nonconjugate braids with equivalent closures, and there are braids with different numbers of strands that have equivalent closures. One specific way to construct braids with different numbers of strands and equivalent closures is through stabilization, illustrated in Figure 4. In 1936 Markov announced (without proof) the following surprising theorem: if two braid closures are equivalent, then, up to conjugacy, the braids differ by a finite sequence of stabilizations, destabilizations, and exchange moves (although it was soon realized that the exchange moves were not needed).

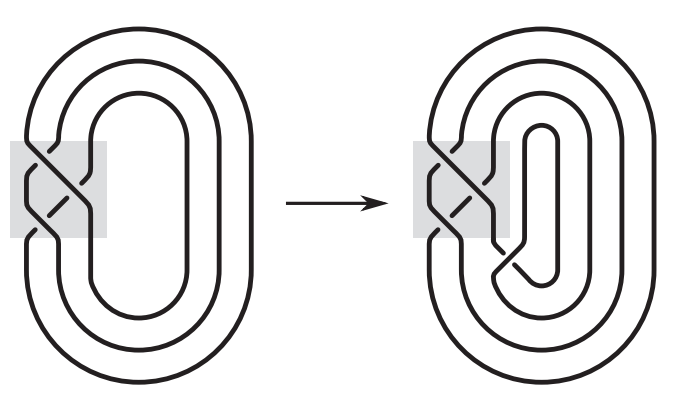

Figure 4. A closed braid and its stabilization.

Four decades later, Birman published a monograph, Braids, links, and mapping class groups [12], based on a graduate course she gave at Princeton University during the academic year 1971-72. Her book was the first comprehensive treatment of braid theory, and its appearance represented the birth of the modern theory. It contains in particular the first complete proof of Markov's theorem.

Our discussion of braids and knots so far points us in three natural directions:

1. the conjugacy problem for the braid group, namely, the problem of algorithmically determining whether or not two elements of $B_{n}$ are conjugate; 
2. the algebraic link problem, namely, the more general problem of algorithmically determining if two braids have equivalent closures; and

3. the big question of whether we can use braid theory to discover new knot invariants.

Birman's monograph focused precisely on these problems. Here we briefly touch on the first two problems, and some contributions to these made by Birman later in her career. In the next section we discuss how the book contributed to the third problem.

With respect to the conjugacy problem, Birman's work has led in two directions. In the 2000s she wrote three papers with Gebhardt and Gonzáles-Meneses [21-23] in which they expand on the Garside approach to the conjugacy problem, explored three decades earlier in Birman's book. A different approach is provided by her paper with Ko and Lee [7]. There, they introduce a new algebraic approach to the braid group, a tool now called the BirmanKo-Lee monoid for the braid group. This is the secondmost cited paper in Birman's catalog.

In the 1990s Birman and Menasco wrote a series of six papers with the title "Studying links via closed braids" [3136]. The fourth in the series was published in Inventiones Mathematicae. A basic question is studied in these papers: If two braids have the same number of strands and have equivalent closures, can we find a sequence of elementary moves that pass from one braid to the other without changing the number of strands? Can we do this algorithmically?

In the end Birman and Menasco did find a "Markov theorem without stabilization," a calculus for dealing with the algebraic link problem [37]. Along the way, they developed connections and applications to the field of contact topology. In particular, they give examples where the isotopy class of a knot and the Bennequin invariant do not fully determine the transverse isotopy class [38]; see also Birman's work with her student Wrinkle [45] as well as the work of Etnyre and Honda [47].

\section{§4 Birman's Book and the Jones Polynomial}

While at Princeton, Birman's research focus was on the third problem described in the last section, namely, using braid theory to discover new knot invariants. One tool that becomes available when we have a group in hand is the subject of representation theory. This is relevant to the theory of knot invariants because conjugacy classes of matrices have many natural invariants, such as the determinant.

At the time of Birman's book, only one interesting representation of the braid group was known, namely, the Burau representation. This representation gives a knot invariant as follows: given a knot, choose a braid whose closure is that knot, apply the Burau representation, subtract this matrix from the identity, take the determinant, and then scale by $(1-t) /\left(1-t^{n}\right)$. This conjugacy class invariant for braids interacts nicely with stabilization, and so we indeed obtain a knot invariant.

The knot invariant arising from the Burau representation turns out to be nothing other than the Alexander polynomial. (To paraphrase one of Birman's sayings, when you discover a new knot invariant, your task is to figure out which existing invariant you have just rediscovered.) The Alexander polynomial is of fundamental importance in knot theory, but as mentioned earlier it is not a complete invariant. And without any new representations on the horizon, it seemed hopeless for Birman to use her ideas to extract knot invariants from braids.

But then in 1984, after Birman became a professor at Columbia University, Vaughan Jones asked to meet with Birman to discuss a new representation of the braid group he had discovered through his work on von Neumann algebras. His representation was a direct sum of matrix representations, one of the summands being the Burau representation. From the representation, Jones extracted a conjugacy class invariant for braids. This was not a determinant (as for the Alexander polynomial) but a weighted sum of the traces of the summands [56].

Birman explained the Markov theorem to Jones, who then realized that his conjugacy invariant for braids gave a new invariant of knots, similar to how the Burau representation gives the Alexander polynomial.

Jones' new polynomial was quickly seen to be an improvement over the Alexander polynomial, as it could distinguish the left- and right-handed trefoil knots. Even better, it evaluated nontrivially on the 11-crossing KinoshitaTerasaka knot [58]. And so the Jones polynomial was born, and a revolution in knot theory was begun.

Jones received the Fields Medal in 1990 for this work. Fittingly, Birman gave the laudation at the International Congress of Mathematicians. See Birman's article from the proceedings [17] and also her personal recollections in this journal [1]. In his Annals paper [57], Jones writes, "The author would like to single out Joan Birman among the many recipients of his thanks. Her contribution to this new topic has been of inestimable importance."

Jones showed that his polynomial is not a complete knot invariant: the Conway knot and the 11-crossing Kinoshita-Terasaka knot have the same Jones polynomial. In a paper published in Inventiones Mathematicae, Birman further found many inequivalent closed 3-braids with the same Jones polynomial [15]. It is an open question whether or not there is a nontrivial knot with trivial Jones polynomial.

Birman and Wenzl used the theory of the Jones polynomial (specifically, the two-variable polynomial of Kauffman) to construct a new representation of the braid group 
[42]. Both Jones and Birman's student Zinno [78] proved that one summand of this representation is the same as the Lawrence representation, famously proved to be faithful by Bigelow [5] and Krammer [61].

Shortly after Jones' discovery, Vassiliev discovered new invariants of knots, named for him (and sometimes called finite-type invariants). Birman and Lin gave a simplified, axiomatic, combinatorial approach to these invariants [29]. This is Birman's most cited paper and was also published in Inventiones Mathematicae. Birman wrote a beautiful survey paper explaining this work and the connection to the Jones polynomial [18]; this article won the Chauvenet Prize in 1996.

\section{$\S 5$ Mapping Class Groups}

We now move on from the world of knots and braids, which are one-dimensional objects, to the realm of surfaces, which are inherently two-dimensional. The theory of mapping class groups of surfaces was initiated by Dehn in the 1920s. Dehn was the doctoral advisor of Magnus who, in turn, was the advisor to Birman. As we will see, mapping class groups will play a prominent role in Birman's career.

To start at the beginning, a surface is a two-dimensional manifold. For each $g \geq 0$ there is a surface $S_{g}$ of genus $g$, obtained as the connect sum of $g$ tori (so $S_{0}$ is the sphere, and $S_{1}$ is the torus). The classification of surfaces says that these are all of the surfaces that are closed (compact and without boundary) and orientable.
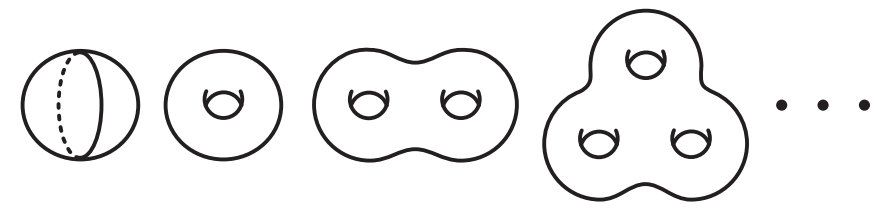

Figure 5. The first few closed, orientable surfaces.

While surfaces are completely classified, there are many open questions, and the theory of surfaces is an active area of research today. Of particular interest is the mapping class group MCG $(S)$ of a surface $S$, the group of homotopy classes of homeomorphisms of $S$. This is a discrete group that encodes the symmetries of $S$. One source of nontrivial elements of MCG $(S)$ is the set of rotations of $S$. For instance the surface $S_{3}$ in Figure 5 admits an obvious rotation of order 3.

An important type of infinite order element is a Dehn twist. In Figure 6 we depict a twist of the annulus. A Dehn twist on a surface is a homeomorphism that performs such a twist on some annulus and is the identity on the complement. If $c$ is a simple closed curve in $S$, then the Dehn twist about an annular neighborhood of $c$ is a well-defined element $T_{C}$ of $\operatorname{MCG}(S)$.

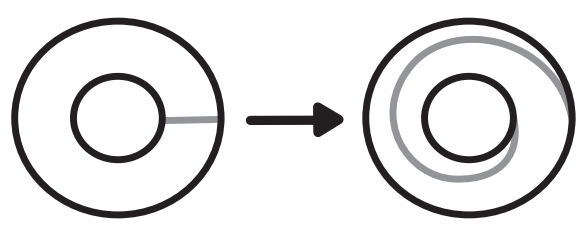

Figure 6. A twist of an annulus.

Dehn proved the foundational theorem that $\operatorname{MCG}\left(S_{g}\right)$ is finitely generated by Dehn twists. Dehn's point of view was motivated by the following analogy:

linear maps : vectors :: mapping classes : curves

More specifically, Dehn was interested in simple closed curves, those with no self-intersections. He referred to the set of these as the arithmetic field of the surface.

After the early work of Dehn and his student Nielsen, the subject of mapping class groups was largely forgotten. Birman reignited interest in the subject through her thesis work (see Section 8, "The Birman Exact Sequence"), her book, and her various survey articles $[13,14,20]$. The subject really exploded with the work of Thurston, which was announced shortly after Birman's book was published; see the next section.

Today, the theory of mapping class groups is a central topic, connected to many fields of mathematics and physics. For instance it can be interpreted as:

1. the outer automorphism group of the fundamental group of the surface;

2. the fundamental group of the moduli space of algebraic curves;

3. the isometry group of Teichmüller space; and

4. the classifying group for surface bundles.

See the primer by Farb and the author [48] for a modern introduction to mapping class groups.

\section{$\S 6$ Curves on Surfaces}

Birman and Series wrote a number of papers aimed at understanding the nature of the set of simple closed curves in a surface. They gave, for instance, an algorithm for determining if an element of the fundamental group of a surface has a simple representative [39]. They also described a sense in which the action of $\operatorname{MCG}(S)$ on the space of simple closed curves in $S$ is linear, as per Dehn's analogy above [41].

The most influential result of Birman and Series [40] addresses the question, What does the set of simple closed curves look like if we draw them all at once? Precisely, they fix a surface of negative Euler characteristic and a hyperbolic metric on the surface, and they consider the (unique) geodesic representative of each homotopy class of simple closed curves. Their main theorem is that the union of all such geodesics is nowhere dense and has Hausdorff dimension 1 . 

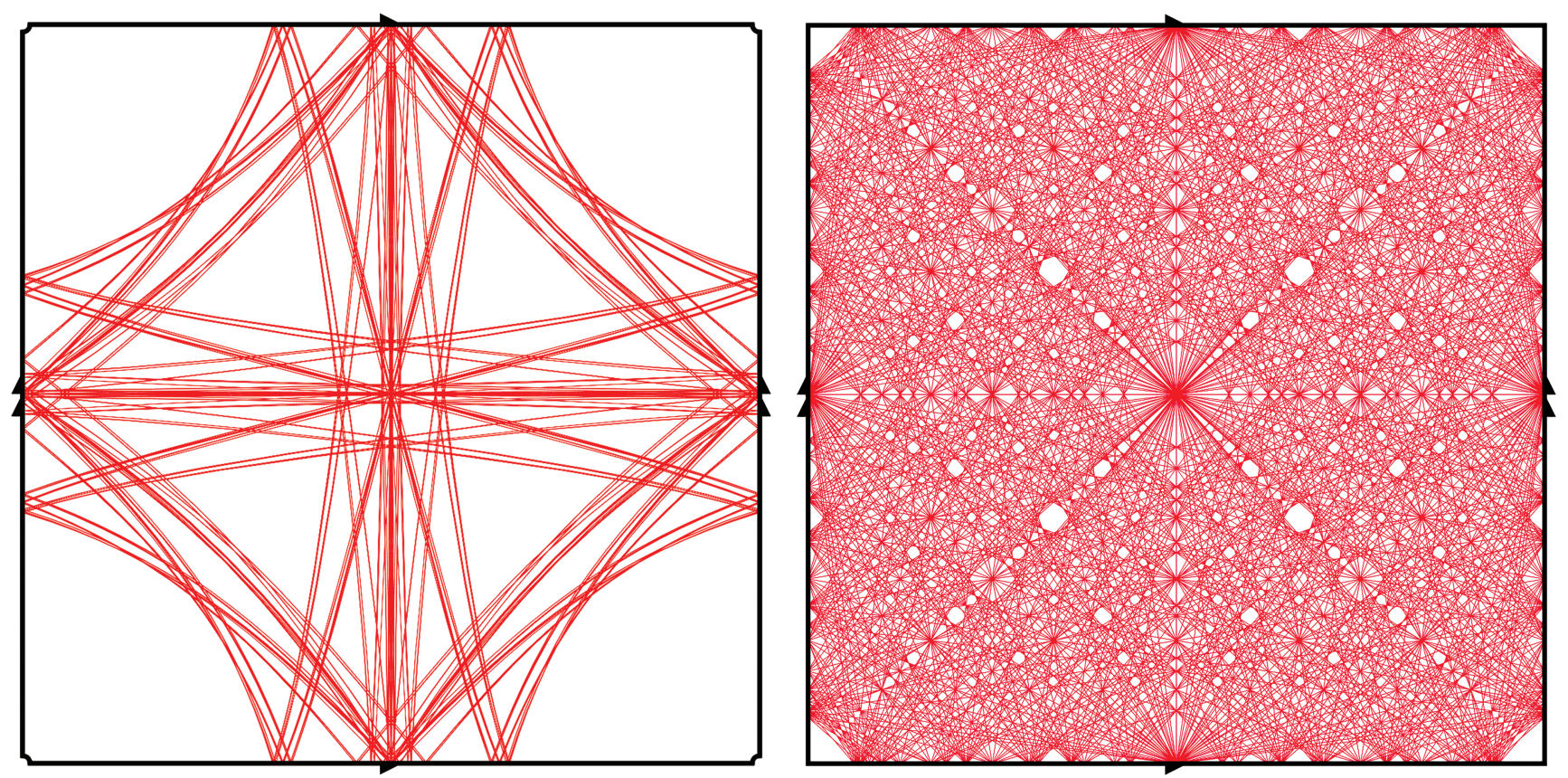

Figure 7. Left(7a): the 88 shortest geodesics on a hyperbolic punctured torus; right(7b): the 88 shortest geodesics on a Euclidean torus.

This result is illustrated by Figure 7. The left side shows a square with the four corners deleted. If we identify opposite sides, we obtain a punctured torus (a torus minus one point). The hyperbolic metric on the latter is mapped to the square by a conformal mapping. Long hyperbolic geodesics are well approximated by arcs of short ones. So even though the picture only shows the 88 shortest simple geodesics, it gives a decent approximation of the union of all simple geodesics.

Here are two striking points of contrast: (1) the union of all closed geodesics (including the ones with self-intersections) is dense; and (2) if we consider a Euclidean torus (the torus obtained by identifying opposite sides of a Euclidean square) and choose one geodesic in each homotopy class of simple closed curves, the resulting union of geodesics is dense (see the right-hand side of Figure 7).

At the end of their paper, Birman and Series suggest another interesting problem: counting the number of simple geodesics as a function of the length. They write:

In fact the degree of the polynomial $P_{0}(n)$ bounding the number of simple geodesics of length $n$ is at most $6 g+2 b-6$, where $g$ is the genus and $b$ the number of boundary components of $M \ldots$ In general the precise nature of the bound seems to be a very interesting number theoretic question.

Many years later, Mirzakhani did find the precise nature of the bound (the upper bound of Birman and Series is also a lower bound), one of the many stunning achievements in her Fields Medal work [68].
The Birman-Series result also plays a central role in the proof of the celebrated McShane identity, which states that for any hyperbolic metric on the punctured torus, we have

$$
\sum_{\gamma} \frac{1}{1+e^{\ell(\gamma)}}=1 / 2,
$$

where the sum is over all simple closed geodesics and $\ell(\gamma)$ denotes the hyperbolic length [66]. This theorem was also generalized by Mirzakhani [67], who used her generalization to compute the volume of moduli space in the WeilPetersson metric.

\section{$\S 7$ Basic Algebraic Properties of the Mapping Class Group}

In this section we discuss Birman's work on the following basic algebraic questions about $\operatorname{MCG}\left(S_{g}\right)$ :

1. What is the abelianization?

2. What is the rank of a maximal torsion-free abelian subgroup?

These are among the first questions we can ask about any infinite group.

Mumford was one of the few mathematicians who studied the mapping class group in the period between Dehn and Birman. He was interested in the applications to algebraic geometry. What he proved [70] is that any abelian quotient of $\operatorname{MCG}\left(S_{g}\right)$ is a quotient of $\mathbb{Z} / 10$ when $g \geq 3$. Birman [11] improved the $\mathbb{Z} / 10$ to $\mathbb{Z} / 2$. Building on this, her student Powell further improved the $\mathbb{Z} / 2$ to the trivial group [73], thus establishing the fundamental theorem 
that $\operatorname{MCG}\left(S_{g}\right)$ is perfect for $g \geq 3$. This completely answers the first question.

The second question was answered in a joint paper by Birman, Lubotzky, and Birman's student McCarthy [30]. The three of them were working to understand Thurston's groundbreaking work on the mapping class group. As a part of his Fields Medal work, Thurston [75] gave a classification of elements of the mapping class group, now called the Nielsen-Thurston classification. This theorem states that every element of the mapping class group has a representative homeomorphism that preserves a (possibly empty) collection of disjoint curves and, on the complementary pieces, is either of finite order or pseudo-Anosov. A pseudo-Anosov map is one that locally looks like the action of the matrix $\left(\begin{array}{cc}\lambda & 0 \\ 0 & \lambda^{-1}\end{array}\right)$ on $\mathbb{R}^{2}$. So there are two invariant foliations, one stretched by $\lambda$ and one by $\lambda^{-1}$.

We should think of Thurston's theorem as a sort of Jordan form for mapping classes. There is one problem: he did not prove that the decomposition along curves was canonical. Birman, Lubotzky, and McCarthy addressed exactly that, by defining the canonical reduction system for a mapping class.

As a result of this work, Birman, Lubotzky, and McCarthy showed that the answer to the second question is $3 g-3$ for $\operatorname{MCG}\left(S_{g}\right)$. They further proved that every solvable subgroup of the mapping class group is virtually abelian.

Like the Jordan canonical form for matrices, canonical reduction systems feature prominently in modern theory of mapping class groups, especially in work on their algebraic structure. For instance, Ivanov and McCarthy used canonical reduction systems to prove that mapping class groups satisfy a Tits alternative, thus strengthening the analogy between mapping class groups and arithmetic groups [51, 65].

\section{\$8 The Birman Exact Sequence}

There are many connections between the theories of braid groups and mapping class groups. The two most important are the Birman exact sequence and the Birman-Hilden theory, discussed in this section and the next. One running theme is that of group presentations for mapping class groups.

Dehn proved that the mapping class group of the torus is isomorphic to $\mathrm{SL}_{2}(\mathbb{Z})$, which has a well-known finite presentation. In her thesis work, Birman's goal was to find group presentations for other mapping class groups. She succeeded right away in finding an inductive procedure for computing presentations of mapping class groups of surfaces with marked points.

Let $S$ be a surface of negative Euler characteristic, and let $p \in S$. We consider $\operatorname{MCG}(S, p)$, the group of homotopy classes of homeomorphisms of $S$ fixing the point $p$ (it is crucial that the homotopies fix $p$ as well). There is a forgetful map $\operatorname{MCG}(S, p) \rightarrow \operatorname{MCG}(S)$. Birman wanted to understand the kernel.

For $[\phi] \in \operatorname{MCG}(S, p)$ to be in the kernel, this means that $\phi$ is homotopic to the identity as long as we allow $p$ to move during the homotopy. If we follow the path of $p$ throughout this homotopy, we obtain a loop in $S$, that is, an element of the fundamental group $\pi_{1}(S, p)$. Birman's theorem is that this identification is well-defined and that it gives an isomorphism of $\pi_{1}(S, p)$ with the kernel.

The resulting map $\pi_{1}(S, p) \rightarrow \operatorname{MCG}(S, p)$ is usually called the push map because we can think of the image of $\alpha \in \pi_{1}(S, p)$ as the element of $\operatorname{MCG}(S, p)$ obtained by pushing $p$ along $\alpha$ (Birman originally called this the spin map).

Birman's result is usually stated as saying that the following sequence is exact:

$$
1 \rightarrow \pi_{1}(S, p) \rightarrow \operatorname{MCG}(S, p) \rightarrow \operatorname{MCG}(S) \rightarrow 1 .
$$

Using this, she could promote a presentation of $\operatorname{MCG}(S)$ to a presentation for $\operatorname{MCG}(S, p)$. The Birman exact sequence is ubiquitous in the theory of mapping class groups, as it is used in many inductive arguments.

What is the connection to braid groups? The first step in this direction is to generalize from one point $p$ to a finite set of points $P=\left\{p_{1}, \ldots, p_{n}\right\}$. The group $\operatorname{MCG}(S, P)$ is the group of homotopy classes of homeomorphisms of $S$ fixing $P$ as a set. Let $C_{n}(S)$ denote the space of configurations of $n$ distinct points in $S$. Birman's more general exact sequence is

$$
1 \rightarrow \pi_{1}\left(C_{n}(S), P\right) \rightarrow \operatorname{MCG}(S, P) \rightarrow \operatorname{MCG}(S) \rightarrow 1 .
$$

When $n=1$, the space $C_{n}(S)$ is homeomorphic to $S$, and so we obtain the first exact sequence above. Recall that $B_{n}$ is defined as $\pi_{1}\left(C_{n}\left(\mathbb{R}^{2}\right), P\right)$. The group $\pi_{1}\left(C_{n}(S), P\right)$ is known as a surface braid group. We can visualize the elements as braided strands in $S \times[0,1]$. As a special case, when $S$ is the disk, we conclude that $B_{n}$ is isomorphic to the mapping class group of a disk with $n$ marked points.

Birman used the more general exact sequence in her thesis to obtain presentations for the mapping class groups of the torus with any number of marked points [10]. The surface of genus 2 would have to wait for her work with Hilden.

\section{§9 The Birman-Hilden Theory}

After graduating from New York University's Courant Institute in 1968, Birman took a job at Stevens Institute of Technology, where she began a very successful collaboration with Hilden, a graduate student there at the time.

Birman and Hilden originally set out to find a presentation for $\operatorname{MCG}\left(S_{2}\right)$, the next natural mountain to climb. The key idea in their work is to relate $\operatorname{MCG}\left(S_{2}\right)$ to a braid group in the following way. The hyperelliptic involution 
$\iota: S_{2} \rightarrow S_{2}$ is the rotation by $\pi$ about the axis indicated in Figure 8.

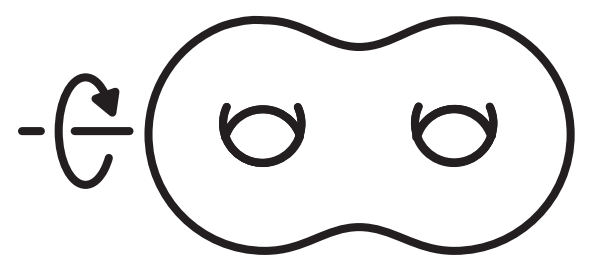

Figure 8. The hyperelliptic involution of $S_{2}$.

The quotient $S_{2} /\langle\iota\rangle$ is a sphere $S_{0,6}$ with six distinguished points (the images of the six fixed points of $\iota$ ). Birman and Hilden proved that there is an isomorphism

$$
\operatorname{MCG}\left(S_{2}\right) /\langle[\iota]\rangle \stackrel{\cong}{M} \operatorname{MCG}\left(S_{0,6}\right) .
$$

Since MCG $\left(S_{0,6}\right)$ is closely related to a braid group (with the sphere replacing the disk), this allowed them to convert a known presentation for $\operatorname{MCG}\left(S_{0,6}\right)$ into a presentation for $\operatorname{MCG}\left(S_{2}\right)$. This work is the subject of Birman's article, "My favorite paper" [9].

The above isomorphism is defined as follows. As observed earlier by Birman, every element of $\operatorname{MCG}\left(S_{2}\right)$ has a representative that commutes with $\iota$. Such a representative descends to a homeomorphism of $S_{0,6}$ and hence gives an element of $\operatorname{MCG}\left(S_{0,6}\right)$. The hard part of their theorem is showing that this map is well-defined, that is, that it interacts well with homotopies.

Birman and Hilden vastly generalized this theorem in a series of papers on hyperelliptic and symmetric mapping class groups [24-26], culminating in their most general result [28], which was published in Annals of Mathematics. This work was later generalized by MacLachlan and Harvey [63] and by Winarski [76], who gave Teichmüller-theoretic and combinatorial-topological points of view.

The Birman-Hilden theory gives a dictionary between the theories of braid groups and mapping class groups, with important applications on both sides. For instance it is used in the proof that $\operatorname{MCG}\left(S_{2}\right)$ is linear $[6,60]$ and also in the resolution of a question of Magnus about the action of the braid group on the fundamental group of the punctured disk [28]. We refer the reader to our survey with Winarski for a detailed discussion [64].

\section{§10 Heegaard Splittings, Torelli Groups, and Homology Spheres}

We now turn to the interface between the theories of surfaces and 3-manifolds. A 3-manifold is the three-dimensiona analogue of a surface, that is, a space that locally looks like $\mathbb{R}^{3}$. A first example is the 3-sphere $S^{3}$. We can use stereographic projection to identify $S^{3}$ as $\mathbb{R}^{3}$ with one added point at infinity, in much the same way that we identify $S^{2}$ as $\mathbb{R}^{2}$ with a point at infinity.
In this section we will focus on one particular construction of 3-manifolds from surfaces, namely Heegaard splittings. If $S_{g}$ is the surface of a donut with $g$ donut holes, then the handlebody $H_{g}$ is the donut itself. By gluing two copies of $H_{g}$ along their boundaries, we obtain a 3manifold without boundary. For each $g$ there is a particular gluing $\psi: S_{g} \rightarrow S_{g}$ that results in the sphere $S^{3}$. (The usual embedding of $H_{g}$ in $\mathbb{R}^{3} \subseteq S^{3}$ is a realization of this gluing: the outside of $H_{g}$ is another copy of $H_{g}$ !) In general, the decomposition of a 3-manifold into two handlebodies glued along their boundary is called a Heegaard splitting.

If we take any homeomorphism $\phi$ of $S_{g}$ and post-compose the gluing map $\psi$ by $\phi$, we obtain a new 3 -manifold. The resulting 3-manifold only depends on the mapping class $[\phi] \in \operatorname{MCG}\left(S_{g}\right)$. What is more, every closed, orientable 3-manifold arises in this way. The upshot is that the theory of Heegaard splittings gives us a set map

$$
\operatorname{MCG}\left(S_{g}\right) \rightarrow \text { 3-manifolds. }
$$

The mapping class group $\operatorname{MCG}\left(S_{g}\right)$ acts on the first homology group $H_{1}\left(S_{g}\right)$. The kernel of this action is called the Torelli group $\mathcal{I}\left(S_{g}\right)$. By the Mayer-Vietoris theorem, we have the restriction

$$
\mathcal{I}\left(S_{g}\right) \rightarrow \text { homology 3-spheres. }
$$

Here, a homology 3-sphere is a 3-manifold that has the same homology groups as $S^{3}$. This is an important subclass of 3-manifolds. Indeed, the fact that there exist nontrivial homology 3-spheres is the reason that the Poincaré conjecture cannot be stated in terms of homology alone (and this is what forced Poincaré to invent $\pi_{1}$ ).

Birman published a number of works on Heegaard splittings, specifically with the aim of classifying 3-manifolds through the lens of the mapping class group. For instance, with Hilden [27] she gave an algorithm to determine if a manifold with a given Heegaard splitting is homeomorphic to $S^{3}$.

\section{§11 Birman's Work on Torelli Groups}

Birman made two monumental contributions to the theory of Torelli groups. In particular, her work was aimed at the following questions:

1. What is a natural generating set for the Torelli group?

2. What are the abelian quotients of the Torelli group?

3. Is the Torelli group finitely generated?

As with mapping class groups, these are among the first properties we would like to know about a group.

There is also a connection with algebraic geometry: the Torelli group encodes the fundamental group of the Torelli space, the space of framed curves of genus $g$. The period 
mapping takes this space to the Siegel upper half-space, sending a framed curve to its period matrix. (Torelli is the name of an Italian algebraic geometer.) As such, the above questions can be reinterpreted as basic questions about the topology of Torelli space.

Birman spent the academic year 1969-70 in Paris. By her own account, she was mathematically isolated there and discouraged [1]. But she had an idea for how to attack the first question by brute-force calculation. The starting point is that the mapping class group $\operatorname{MCG}\left(S_{g}\right)$ and the Torelli group $\mathcal{I}\left(S_{g}\right)$ fit into a short exact sequence

$$
1 \rightarrow \mathcal{I}\left(S_{g}\right) \rightarrow \operatorname{MCG}\left(S_{g}\right) \rightarrow \operatorname{Sp}_{2 g}(\mathbb{Z}) \rightarrow 1 .
$$

The group $\mathrm{Sp}_{2 g}(\mathbb{Z})$ is isomorphic to the automorphism group of $H_{1}\left(S_{g} ; \mathbb{Z}\right) \cong \mathbb{Z}^{2 g}$; we have the symplectic group here instead of the whole general linear group because automorphisms preserve the algebraic intersection form, which is symplectic. From this point of view, we can think of $\mathrm{Sp}_{2 g}(\mathbb{Z})$ as capturing the linear, easy-to-understand aspects of $\operatorname{MCG}\left(S_{g}\right)$ and of $\mathcal{I}\left(S_{g}\right)$ as encapsulating the more difficult, mysterious aspects.

Birman knew that the defining relations for $\operatorname{Sp}_{2 g}(\mathbb{Z})$ correspond to generators for $\mathcal{I}\left(S_{g}\right)$ (this is a general principle that applies to any short exact sequence of groups). So the task then was to find a reasonable group presentation for $\mathrm{Sp}_{2 g}(\mathbb{Z})$. She succeeded and obtained a presentation with three families of generators and 10 families of relations.

Birman's student Powell then gave simple descriptions of the resulting generators for $\mathcal{I}\left(S_{g}\right)$ : they are Dehn twists about separating curves and bounding pair maps [73]. A bounding pair map is $T_{a} T_{b}^{-1}$, where $a$ and $b$ are disjoint, homologous, nonseparating curves; see Figure 9. Putman, who gave a geometric proof of the Birman-Powell result in his thesis [74], describes Birman's work as "absolutely heroic."
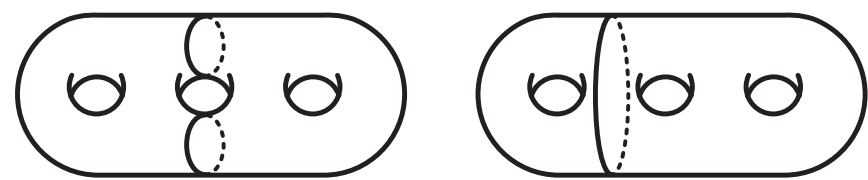

Figure 9. Left: a bounding pair; right: a separating curve.

Birman and Craggs took aim at the second and third questions, and they made a most spectacular contribution. They showed that, unlike $\operatorname{MCG}\left(S_{g}\right)$, the group $\mathcal{I}\left(S_{g}\right)$ does have nontrivial abelian quotients. They found a family of homomorphisms $\rho_{\psi}: \mathcal{I}\left(S_{g}\right) \rightarrow \mathbb{Z} / 2$. Surprisingly, the definition involves the theories of 3- and 4-manifolds. One hope they had was that there would be infinitely many distinct such homomorphisms, thus proving that $\mathcal{I}\left(S_{g}\right)$ was not finitely generated.
In order to specify one of the Birman-Craggs homomorphisms, we need to fix some Heegaard splitting $\psi$ of $S^{3}$. Now let $f \in \mathcal{I}\left(S_{g}\right)$. As in Section 10, "Heegaard Splittings, Torelli Groups, and Homology Spheres," $f$ determines a homology 3-sphere $M_{f}$. Every homology 3-sphere is the boundary of some 4-manifold. The Rokhlin invariant of $M_{f}$ is the signature of this 4-manifold, divided by $8, \bmod 2$ (by Rokhlin's theorem, this is well-defined). This element of $\mathbb{Z} / 2$ is $\rho_{\psi}(f)$. Miraculously, this defines a homomorphism $\mathcal{I}\left(S_{g}\right) \rightarrow \mathbb{Z} / 2$. The proof features what is probably the first instance of a 4-manifold trisection, a tool popularized four decades later by David Gay and Robion Kirby [79].

Several years after these works, Johnson arrived on the scene. In a stunning series of deep, beautiful papers, he expanded on the work of Birman and her collaborators. He proved [55] that $\mathcal{I}\left(S_{g}\right)$ is finitely generated for $g \geq 3$. Also he classified the Birman-Craggs homomorphismsshowing directly that there were only finitely many-and gave a complete description of the abelianization of $\mathcal{I}\left(S_{g}\right)$ [52]. (Amazingly, there is still no definition of these homomorphisms that does not involve the construction of a 4-manifold.) As a byproduct, Johnson showed that $\mathcal{I}\left(S_{g}\right)$ cannot be generated by Dehn twists about separating curves, disproving a conjecture of Birman.

See Johnson's delightful survey for more about his work [53]. In the survey, Johnson notes that the interest in Torelli groups from topologists "was initiated principally through the work of Joan Birman" [54].

\section{§12 Lorenz Knots}

We end by discussing the work of Birman and Williams on Lorenz knots in the early 1980s. This is a fitting finale, as it combines all four of the main objects of study in this article. It is also a prime example of work that was ahead of its time, with 94 of its 106 citations on MathSciNet ${ }^{\circledR}$ coming after the year 2000.

E. N. Lorenz was a pioneer of chaos theory. He was particularly interested in the weather, and whether it was deterministic. Lorenz is perhaps most famous for coining the phrase "butterfly effect."

In order to help understand weather patterns, Lorenz devised a simplified version of the Navier-Stokes equations, a system of three ordinary differential equations in three variables [62]. This system has a strange attractor, called the Lorenz attractor, shown in the top of Figure 10. Forward trajectories of points converge to the attractor and, once there, stay forever.

A Lorenz knot is a knot obtained as a periodic orbit in the Lorenz attractor. Williams showed that Lorenz knots are exactly the ones that can be drawn on the "template" shown at the bottom of Figure 10.

A Lorenz braid is a braid consisting of strands that either go monotonically left to right or from right to left, where 


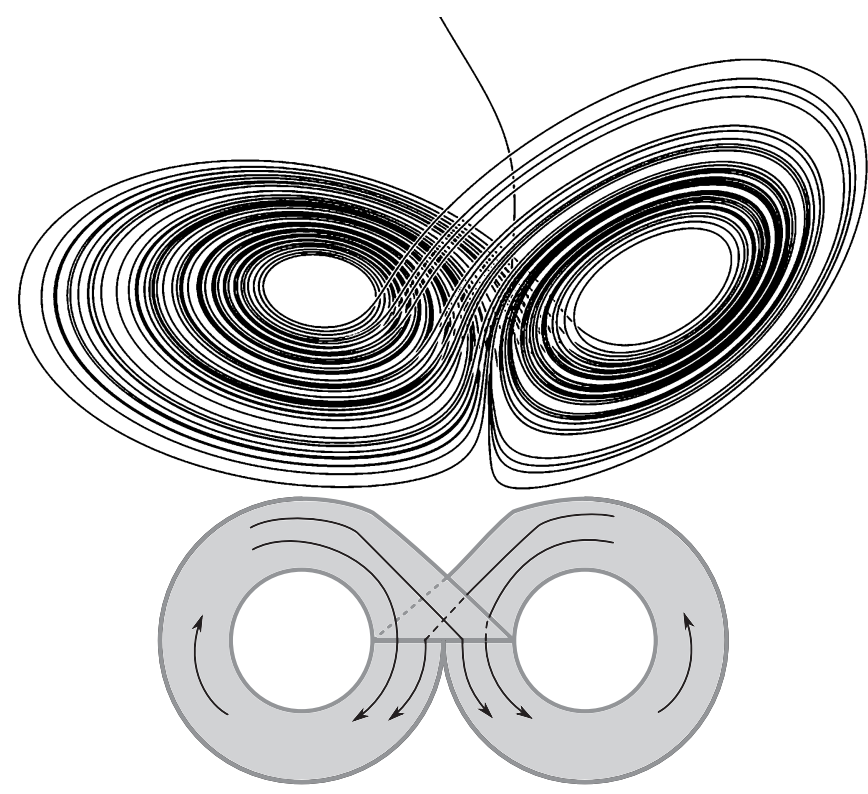

Figure 10. Top(10a): the Lorenz attractor; bottom(10b): the Lorenz template.

the strands going from left to right pass over the strands going from right to left, and where neither the left-to-right nor the right-to-left strands cross amongst themselves; see Figure 11. Lorenz knots can also be described as the closures of Lorenz braids.

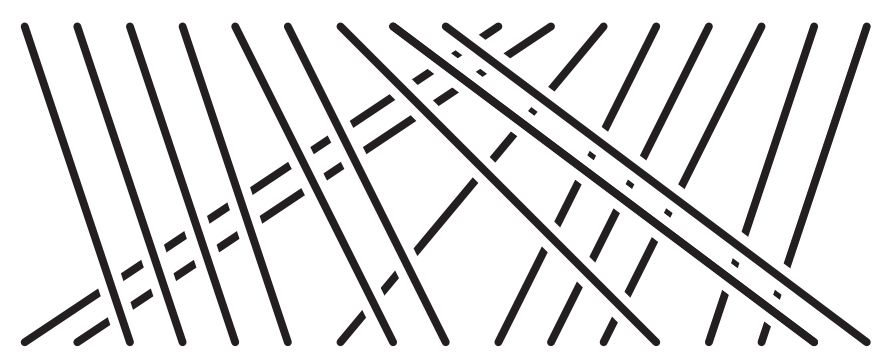

Figure 11. A Lorenz braid.

Williams approached Birman at a conference and asked her if she could identify some of the knots he was studying. She could, and their discussion quickly turned into a fruitful collaboration. In their first paper [44], Birman and Williams proved many theorems about Lorenz knots, including:

1. There are infinitely many (inequivalent) Lorenz knots.

2. Lorenz knots are prime.

3. Every algebraic knot is a Lorenz knot.

4. Every Lorenz knot is fibered.

In the third theorem, an algebraic knot is any component of the link of an isolated singularity of a complex curve. The fourth theorem requires some explanation. We can construct a 3-manifold from a surface $S$ by the mapping torus construction: for $[\phi] \in \operatorname{MCG}(S)$, we take the product $S \times[0,1]$ and glue $S \times\{0\}$ to $S \times\{1\}$ by $\phi$. The resulting 3-manifold has a natural map to $S^{1}$ with fiber $S$, and we say that the 3 -manifold is fibered. A knot in $\mathbb{R}^{3}$ is said to be fibered if its complement in $S^{3}$ is a fibered 3-manifold.

Two decades after Birman and Williams, Ghys entered the picture. He was studying the manifold $M=\mathrm{PSL}_{2}(\mathbb{R}) /$ $\mathrm{PSL}_{2}(\mathbb{Z})$. The manifold $M$ is homeomorphic to the complement in $S^{3}$ of the trefoil knot, and it can also be described as the unit tangent bundle of the modular surface (the quotient of the hyperbolic plane by $\mathrm{PSL}_{2}(\mathbb{Z})$ ). From the latter description, $M$ has a geodesic flow. Ghys was studying the closed orbits in this flow, and he proved that the knots arising from these closed orbits are in natural bijection with the Lorenz knots (the connection was further investigated by Pinsky [72]). He further showed that the Rademacher function exactly records the linking number of each knot with the missing trefoil. We recommend Ghys's beautiful survey, written on the occasion of his plenary lecture at the International Congress of Mathematicians [50].

We next turn to the question, How common are Lorenz knots? Dehornoy, Ghys, and Jablon showed that of the $1,701,936$ knots with at most 16 crossings in their diagrams, only 20 are Lorenz knots. And so from this point of view they appear to be rather rare. Birman and her postdoc Kofman took a different point of view. In order to explain it, we take a detour into hyperbolic geometry and the classification of 3-manifolds.

Thurston revolutionized the theory of 3-manifolds by showing that many knots are hyperbolic; that is, their complements in $S^{3}$ could be given complete Riemannian metrics of constant sectional curvature -1 . By the Mostow rigidity theorem, hyperbolic structures on 3-manifolds are unique. In particular, a hyperbolic knot has a well-defined volume.

Thurston's work on knots eventually led him to formulate his geometrization conjecture, which shaped the field for several decades. The conjecture states that every 3-manifold can be decomposed into geometric pieces, namely, Seifert-fibered spaces (completely classified in the 1930s by Seifert) and hyperbolic manifolds. The Poincaré conjecture is a special case of Thurston's conjecture because there are no counterexamples to the latter among the Seifertfiber

-ed spaces or the closed hyperbolic manifolds (which have infinite fundamental group).

The geometrization conjecture was famously proved by Perelman in 2003; see [46,59,69]. More recently, Agol and Wise proved that every closed hyperbolic 3-manifold has a finite cover that is fibered, verifying another conjecture of Thurston $[2,4,77]$. This gives a satisfying description of 
the hyperbolic pieces of a 3-manifold: up to taking finite covers, they all come from surface homeomorphisms.

We return now to our story about Lorenz knots. Rather than organizing knots by the number of crossings in their diagrams, Birman and Kofman organized the hyperbolic knots by their volumes. They showed that of the 201 hyperbolic knots of smallest volume, more than half of them are Lorenz knots [8]. So among all knots, Lorenz knots are extremely rare, but among the small-volume hyperbolic knots, Lorenz knots are quite prevalent.

Birman and Williams wrote a companion paper [43] where they studied a different flow on $S^{3}$ and discovered an appropriate template in that case as well. In his gem of a thesis, Ghrist [49] showed that this flow is universal, in that it contains all knots as closed orbits, disproving a conjecture of Birman and Williams.

There are many other intriguing aspects to the story and tantalizing questions to answer. As Birman writes at the end of her survey [19], "There is a big world out there, and a great deal of structure, waiting to be discovered!"

\section{Epilogue}

A distinguishing feature of Birman's career is that her research has been motivated by her own vision, interests, and curiosity. There are very few instances where Birman was trying to answer someone else's question or solve someone else's problem. While this may seem like a risky way to approach a career in mathematics, it is hard to argue with the results. Besides the beautiful mathematics she has produced by herself and with her collaborators, she has had (as we have seen) a direct impact on two Fields Medals (Jones' and Mirzakhani's) and a plenary address at the International Congress of Mathematicians (Ghys'), among the many works she has helped to inspire.

As we touched on at the outset and throughout this article, Birman's work was in many cases ahead of its time, her foundational work finding applications (and appreciation) many years after the original discovery. Braid groups, mapping class groups, Torelli groups, and Lorenz knots were fringe topics when she started. With the breakthroughs of Jones, Mirzakhani, Thurston, Johnson, and Ghys we have seen the impact and validation of Birman's work.

As a recent collaborator of Birman's and as a researcher in the same field, the author has had the pleasure of seeing Birman's mathematics from up close and being inspired by her work. We eagerly look forward to the next chapters of Birman's career, including new discoveries by Birman herself and new perspectives on her prior work, yet to be uncovered.

\section{References}

[1] Interview with Joan Birman. Notices of the American Mathematical Society, 54(1):20-29, 2007. MR2275922

[2] Agol I. The virtual Haken conjecture. Doc. Math., 18:1045-1087, 2013. With an appendix by Agol, Daniel Groves, and Jason Manning. MR3104553

[3] Alexander J. A lemma on systems of knotted curves. Proc. Natl. Acad. Sci. USA, 9:93-95, 1923.

[4] Bestvina M. Geometric group theory and 3-manifolds hand in hand: the fulfillment of Thurston's vision. Bull. Amer. Math. Soc. (N.S.), 51(1):53-70, 2014. MR3119822

[5] Bigelow SJ. Braid groups are linear. J. Amer. Math. Soc., 14(2):471-486, 2001. MR1815219

[6] Bigelow SJ and Budney RD. The mapping class group of a genus two surface is linear. Algebr. Geom. Topol., 1:699-708, 2001. MR1875613

[7] Birman J, Ko KH, and Lee SJ. A new approach to the word and conjugacy problems in the braid groups. Adv. Math., 139(2):322-353, 1998. MR1654165

[8] Birman J and Kofman I. A new twist on Lorenz links. J. Topol., 2(2):227-248, 2009. MR2529294

[9] Birman JS. My favorite paper. Celebratio Mathematica, (to appear). MR1873103

[10] Birman JS. Mapping class groups and their relationship to braid groups. Comm. Pure Appl. Math., 22:213-238, 1969. MR0243519

[11] Birman JS. Abelian quotients of the mapping class group of a 2-manifold. Bull. Amer. Math. Soc., 76:147-150, 1970. MR0249603

[12] Birman JS. Braids, links, and mapping class groups. Annals of Mathematics Studies, No. 82, Princeton University Press, Princeton, N.J.; University of Tokyo Press, Tokyo, 1974. MR0375281

[13] Birman JS. Mapping class groups of surfaces: a survey. Discontinuous groups and Riemann surfaces (Proc. Conf., Univ. Maryland, College Park, Md., 1973), pp. 57-71. Ann. of Math. Studies, No. 79, 1974. MR0380762

[14] Birman JS. The algebraic structure of surface mapping class groups. Discrete groups and automorphic functions (Proc. Conf., Cambridge, 1975), pp. 163-198, 1977. MR0488019

[15] Birman JS. On the Jones polynomial of closed 3-braids. Invent. Math., 81(2):287-294, 1985. MR799267

[16] Birman JS. Mapping class groups of surfaces. In Braids (Santa Cruz, CA, 1986), pp. 13-43, Contemp. Math., 78, Amer. Math. Soc., Providence, RI, 1988. MR975076

[17] Birman JS. The work of Vaughan F. R. Jones. In Proceedings of the International Congress of Mathematicians, Vol. I, II (Kyoto, 1990), 9-18. Math. Soc. Japan, Tokyo, 1991. MR1159199

[18] Birman JS. New points of view in knot theory. Bull. Amer. Math. Soc. (N.S.), 28(2):253-287, 1993. MR1191478

[19] Birman JS. The mathematics of Lorenz knots. In Topology and dynamics of chaos, pp. 127-148, World Sci. Ser. Nonlinear Sci. Ser. A Monogr. Treatises, 84, World Sci. Publ., Hackensack, NJ, 2013. MR3289735

[20] Birman JS and Brendle TE. Braids: a survey. In Handbook of knot theory, pp. 19-103. Elsevier B. V., Amsterdam, 2005. MR2179260 
[21] Birman JS, Gebhardt V, and González-Meneses J. Conjugacy in Garside groups. I. Cyclings, powers and rigidity. Groups Geom. Dyn., 1(3):221-279, 2007. MR2314045

[22] Birman JS, Gebhardt V, and González-Meneses J. Conjugacy in Garside groups. III. Periodic braids. J. Algebra, 316(2):746-776, 2007. MR2358613

[23] Birman JS, Gebhardt V, and González-Meneses J. Conjugacy in Garside groups. II. Structure of the ultra summit set. Groups Geom. Dyn., 2(1):13-61, 2008. MR2367207

[24] Birman JS and Hilden HM. On the mapping class groups of closed surfaces as covering spaces. Advances in the theory of Riemann surfaces (Proc. Conf., Stony Brook, NY, $1969)$ pp. 81-115. Ann. of Math. Studies, No. 66, Princeton Univ. Press, Princeton, NJ, 1971. MR0292082

[25] Birman JS and Hilden HM. Isotopies of homeomorphisms of Riemann surfaces and a theorem about Artin's braid group. Bull. Amer. Math. Soc., 78:1002-1004, 1972. MR0307217

[26] Birman JS and Hilden HM. Lifting and projecting homeomorphisms. Arch. Math. (Basel), 23:428-434, 1972. MR0321071

[27] Birman JS and Hilden HM. The homeomorphism problem for $S^{3}$. Bull. Amer. Math. Soc., 79:1006-1010, 1973. MR0319180

[28] Birman JS and Hilden HM. On isotopies of homeomorphisms of Riemann surfaces. Ann. of Math. (2), 97:424439, 1973. MR0325959

[29] Birman JS and Lin X-S. Knot polynomials and Vassiliev's invariants. Invent. Math., 111(2):225-270, 1993. MR1198809

[30] Birman JS, Lubotzky A, and McCarthy J. Abelian and solvable subgroups of the mapping class groups. Duke Math. J., 50(4):1107-1120, 1983. MR726319

[31] Birman JS and Menasco WW. Studying links via closed braids. IV. Composite links and split links. Invent. Math., 102(1):115-139, 1990. MR1069243

[32] Birman JS and Menasco WW. Studying links via closed braids. II. On a theorem of Bennequin. Topology Appl., 40(1):71-82, 1991. MR1114092

[33] Birman JS and Menasco WW. Studying links via closed braids. I. A finiteness theorem. Pacific J. Math., 154(1):1736, 1992. MR1154731

[34] Birman JS and Menasco WW. Studying links via closed braids. V. The unlink. Trans. Amer. Math. Soc., 329(2):585606, 1992. MR1030509

[35] Birman JS and Menasco WW. Studying links via closed braids. VI. A nonfiniteness theorem. Pacific J. Math., 156(2):265-285, 1992. MR1186805

[36] Birman JS and Menasco WW. Studying links via closed braids. III. Classifying links which are closed 3-braids. $\mathrm{Pa}$ cific J. Math., 161(1):25-113, 1993. MR1237139

[37] Birman JS and Menasco WW. Stabilization in the braid groups. I. MTWS. Geom. Topol., 10:413-540, 2006. MR2224463

[38] Birman JS and Menasco WW. Stabilization in the braid groups. II. Transversal simplicity of knots. Geom. Topol., 10:1425-1452, 2006. MR2255503
[39] Birman JS and Series C. An algorithm for simple curves on surfaces. J. London Math. Soc. (2), 29(2):331-342, 1984. MR744104

[40] Birman JS and Series C. Geodesics with bounded intersection number on surfaces are sparsely distributed. Topology, 24(2):217-225, 1985. MR793185

[41] Birman JS and Series C. Algebraic linearity for an automorphism of a surface group. J. Pure Appl. Algebra, 52(3):227-275, 1988. MR952081

[42] Birman JS and Wenzl H. Braids, link polynomials and a new algebra. Trans. Amer. Math. Soc., 313(1):249-273, 1989. MR992598

[43] Birman JS and Williams RF. Knotted periodic orbits in dynamical system. II. Knot holders for fibered knots. In Low-dimensional topology (San Francisco, Calif., 1981), pp. 1-60 Contemp. Math., 120, Amer. Math. Soc., Providence, RI, 1983. MR718132

[44] Birman JS and Williams RF. Knotted periodic orbits in dynamical systems. I. Lorenz's equations. Topology, 22(1):47-82, 1983. MR682059

[45] Birman JS and Wrinkle NC. On transversally simple knots. J. Differential Geom., 55(2):325-354, 2000. MR1847313

[46] Cao H-D and Zhu X-P. A complete proof of the Poincaré and geometrization conjectures-application of the Hamilton-Perelman theory of the Ricci flow. Asian J. Math., 10(2):165-492, 2006. MR2233789

[47] Etnyre JB and Honda K. Cabling and transverse simplicity. Ann. of Math. (2), 162(3):1305-1333, 2005. MR2179731

[48] Farb B and Margalit D. A Primer on Mapping Class Groups. Princeton Mathematical Series, 49. Princeton University Press, 2012. MR2850125

[49] Ghrist RW. Flows on $S^{3}$ supporting all links as orbits. Electron. Res. Announc. Amer. Math. Soc., 1(2):91-97, 1995. MR1350685

[50] Ghys E. Knots and dynamics. In International Congress of Mathematicians. Vol. I, 247-277. Eur. Math. Soc., Zürich, 2007. MR2334193

[51] Ivanov NV. Algebraic properties of the Teichmüller modular group. Dokl. Akad. Nauk SSSR, 275(4):786-789, 1984. MR745513

[52] Johnson D. The structure of the Torelli group. I. A finite set of generators for $\mathcal{I}$. Ann. of Math. (2), 118(3):423-442, 1983. MR727699

[53] Johnson D. A survey of the Torelli group. In Lowdimensional topology (San Francisco, Calif., 1981), pp. 165-179, Contemp. Math., 20, Amer. Math. Soc., Providence, RI, 1983. MR718141

[54] Johnson D. The structure of the Torelli group. II. A chracterization of the group generated by twists on bounding curves.Topology 24(2):113-126, 1985. MR0793178

[55] Johnson D. The structure of the Torelli group. III. The abelianization of $\mathcal{T}$. Topology, 24(2):127-144, 1985. MR793179

[56] Jones VFR. Index for subfactors. Invent. Math., 72(1):125, 1983. MR696688 
[57] Jones VFR. Hecke algebra representations of braid groups and link polynomials. Ann. of Math. (2), 126(2):335-388, 1987. MR908150

[58] Jones VFR. A polynomial invariant for knots via von Neumann algebras. Bull. Amer. Math. Soc. (N.S.), 12(1):103-111, 1985. MR766964

[59] Kleiner B and Lott J. Notes on Perelman's papers. Geometry \& Topology, 12(5):2587-2855, Nov 2008. MR2460872

[60] Korkmaz M. On the linearity of certain mapping class groups. Turkish J. Math., 24(4):367-371, 2000. MR1803819

[61] Krammer D. Braid groups are linear. Ann. of Math. (2), 155(1):131-156, 2002. MR1888796

[62] Lorenz EN. Deterministic, non-periodic flows. J. Atmos. Sci., 20:130-141, 1963.

[63] Maclachlan C and Harvey WJ. On mapping-class groups and Teichmüller spaces. Proc. London Math. Soc. (3), 30(part 4):496-512, 1975. MR0374414

[64] Margalit D and Winarski R. The Birman-Hilden theory. preprint.

[65] McCarthy J. A "Tits-alternative" for subgroups of surface mapping class groups. Trans. Amer. Math. Soc., 291(2):583612, 1985. MR800253

[66] McShane G. Simple geodesics and a series constant over Teichmuller space. Invent. Math., 132(3):607-632, 1998. MR1625712

[67] Mirzakhani M. Weil-Petersson volumes and intersection theory on the moduli space of curves. J. Amer. Math. Soc., 20(1):1-23, 2007. MR2257394

[68] Mirzakhani M. Growth of the number of simple closed geodesics on hyperbolic surfaces. Ann. of Math. (2), 168(1):97-125, 2008. MR2415399

[69] Morgan J and Tian G. Ricci flow and the Poincaré conjecture, Clay Mathematics Monographs 3, American Mathematical Society, Providence, RI; Clay Mathematics Institute, Cambridge, MA, 2007. MR2334563

[70] Mumford D. Abelian quotients of the Teichmüller modular group. J. Analyse Math., 18:227-244, 1967. MR0219543

[71] Murasugi K. Knot theory and its applications. Birkhäuser Boston, Inc., Boston, MA, 1996. Translated from the 1993 Japanese original by Bohdan Kurpita. MR1391727

[72] Pinsky T. On the topology of the Lorenz system. Proc. A., 473(2205):20170374, 11, 2017. MR3710339

[73] Powell J. Two theorems on the mapping class group of a surface. Proc. Amer. Math. Soc., 68(3):347-350, 1978. MR0494115

[74] Putman A. Cutting and pasting in the Torelli group. Geom. Topol., 11:829-865, 2007. MR2302503

[75] Thurston WP. On the geometry and dynamics of diffeomorphisms of surfaces. Bull. Amer. Math. Soc. (N.S.), 19(2):417-431, 1988. MR956596

[76] Winarski RR. Symmetry, isotopy, and irregular covers. Geom. Dedicata, 177:213-227, 2015. MR3370031

[77] Wise DT. From riches to raags: 3-manifolds, right-angled Artin groups, and cubical geometry, CBMS Regional Conference Series in Mathematics, 117. Published for the Conference Board of the Mathematical Sciences, Washington, DC; by the American Mathematical Society, Providence, RI, 2012. MR2986461

[78] Zinno MG. On Krammer's representation of the braid group. Math. Ann., 321(1):197-211, 2001. MR1857374

[79] Gay D and Kirby R. Trisecting 4-manifolds. Geom. Topol., 20 (6):3097-3132, 2016. MR3590351

ACKNOWLEDGMENT. We would like to thank Joan Birman for her help in preparing this article, Shane Scott for making the figures, and Tara Brendle, Lei Chen, Benson Farb, Vaughan Jones, Justin Lanier, Kevin Wortman, and an anonymous referee for helpful conversations.

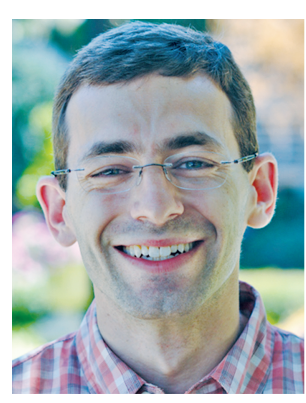

Dan Margalit

Credits

The opening page figure is by David Dumas

(see dumas . io/bi rmanseries/).

Figures $1-6 ; 8-9 ; 10 \mathrm{~b}$ and 11 are by Shane Scott.

Figure 7 is by David Dumas

(see dumas . io/bi rmanseries/).

Figure 10a is by Thierry Dugnolle [CC BY-SA 4.0

(creat i vecommons . org/1 i censes/by-sa/4 .0)], from

Wikimedia Commons.

Author photo courtesy of Joseph Rabinoff. 